\title{
Research Paper \\ Construction and Validation the Healthy Lifestyle Questionnaire Among the Iranian Elderly Who Receive Home Care Services
}

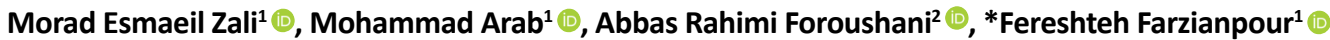

1. Department of Health Management and Economics, School of Public Health, Tehran University of Medical Sciences, Tehran, Iran.

2. Department of Epidemiology and Biostatistics, School of Public Health, Tehran University of Medical Sciences, Tehran, Iran.

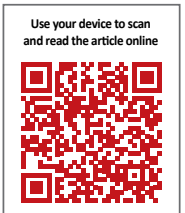

dtation: Esmaeil Zali M, Arab M, Rahimi Foroushani A, Farzianpour F. [Construction and Validation the Healthy Lifestyle Questionnaire Among the Iranian Elderly Who Receive Home Care Services (Persian)]. Iranian Journal of Ageing. 2020; 15(2):130-143. https://doi.org/10.32598/sija.2020.3.180

Key words: Health, Lifestyle, Questionnaire, Validity and reliability, Elderly

\begin{abstract}
Objectives Investigating the elderly's lifestyle conditions is a challenging issue, due to various factors influencing lifestyle. The current study was developed to explore the construct and validity of the Healthy Lifestyle Questionnaire (HLQ) among the Iranian elderly who receive home care services.

Methods \& Materials This combinatory, sequential exploratory study was conducted for creating a lifestyle measuring tool among the elderly in Tehran City, Iran, in 2018. The research was performed in 4 steps including the following: studying semi-structured texts and interviews (the elderly \& specialists); extracting fields and factors related to lifestyle; validity examination, and the final assessment on the target community (reliability). To check the validity, ratio tests were implemented by the expert's panel ( $n=20$ in 8 different specialty fields related to the elderly's treatment). Besides, Cronbach's alpha coefficient was implemented to check the scale's reliability ( $n=40)$. SPSS was also used for the statistical analysis of the collected data. Results The agreed limit for the Content Validity Ratio (CVR) was selected as $42 \%$ according to the specialist's number. Accordingly, out of 130 extracted questions, only 69 questions, CVR point was more than the agreed limit ( $C V R \geq 0.42)$. Thus, the remained question's CVR point equaled 52.5 , by dividing it by the number of remained questions. CVI was calculated to be 0.76 for the whole questionnaire. Moreover, CVI was more than the agreed limit for all the questions in 11 fields $(C V I \geq 0.76)$. Lifestyle's Cronbach's alpha coefficient (0.979) was $>0.7$.

Conclusion The Elderly's Healthy Lifestyle Questionnaire (EHLSQ) has appropriate reliability and validity for assessing lifestyle in the elderly. It can be used to evaluate lifestyle among the elderly in various studies.
\end{abstract}

\section{Extended Abstract}

\section{Introduction}

$\mathrm{T}$

he elderly issues have changed into one of the most challenging and essential topics. This is due to the increased life expectancy, decreased fertility, decreased birth rate, healthcare improvement, and increased el- the elderly population will have reached one billion and 200 individuals (14\% of the world population) [3]; therefore, pursuing healthy aging, exposure of a high proportion of the population to certain diseases of this period is inevitable and requires extensive health service. Due to aging, the individuals' health will deteriorate and the number of diseases will rise [4]. Iran, as a developing country, has a young population; soon, this population will move to the upper part of the population pyramid [5]. Lifestyle is a manner by which individuals' health, welfare, and life quality are provided,

derly population. According to the estimations, by 2025 ,

* Corresponding Author:

Fereshteh Farzianpour

Address: Department of Health Management and Economics, School of Public Health, Tehran University of Medical Sciences, Tehran, Iran.

Tel: +98 (912) 1481269

E-mail: farzianp@sina.tums.ac.ir 
maintained, and improved [6]. Furthermore, lifestyle refers to behavior patterns related to health, i.e. according to the individuals' accessible life opportunities [7, 8]. Based on studies, there is an increase in the effective criteria on lifestyle and the lack of proper and comprehensive scales about the elderly's healthy lifestyle in Iran.

The present study intended to design and validate a healthy lifestyle questionnaire among the Iranian elderly. This goal was achieved by comprehensively reviewing the previous studies and benefiting from the elderly's ideas and experiences, as well as the experts of elderly issues.

The present study aimed to develop a comprehensive scale that includes all aspects of lifestyle proportionate to its theoretical concept.

\section{Methods \& Materials}

The present study applied a combined method; consecutive exploratory aiming at generating lifestyle assessment scale among the elderly living in Tehran City, Iran, in 2018. The study was conducted in 4 steps, as follows: reviewing texts and semi-structured interviews (the elderly \& experts); extracting domains and items related to lifestyle; performing validity examination, and conducting a final assessment on the target society (reliability check). To evaluate the validity, Content Validity Index (CVI) and Content Validity Ratio (CVR) were implemented by the expert's panel ( $n=20$ in 8 different specialties related to the elderly's healthcare issues). Moreover, to assess its reliability, Cronbach's alpha coefficient $(\mathrm{n}=40)$ was implemented. The statistical analysis was conducted by SPSS.

\section{Results}

The agreed unit for CVR was calculated as 42 according to the experts' numbers. CVR value was calculated for each question in different fields.

Table 1 presents the achieved CVI and CVR scores of every question for each field. The questions with scores less than the agreed limit were eliminated $(\mathrm{CVR} \leq 0.76)$; accordingly, out of 130 extracted questions, the CVR was above the agreed limit for 69 questions (CVR $\geq 0.42$ ). CVI was calculated to be 0.76 for the whole questionnaire. In 11 fields, CVI exceeded the agreed limit for all questions $(\mathrm{CVR} \leq 0.76)$. Cronbach's alpha coefficient equaled $>0.7$ in all defined fields.

\section{Conclusion}

The designed questionnaire in the present study (EHLSQ), compared with other questionnaires, not only covers more aspects of the elderly's lifestyle, but it can also be used to assess all the elderly's lifestyle; it is not limited to the elderly with special conditions. Therefore, it has the necessary comprehensiveness to assess all elderly's lifestyle. Moreover, the researchers benefited from the elderly's ideas while interviewing them in designing the EHLSQ. Furthermore, the EHLSQ provided a desirable validity and reliability due to benefiting from more experts' opinions (20 individuals); diversity in the specialty (8 specialties); CVR and CVI assessment; using the target group opinions (the elderly), and obtaining a high Cronbach's alpha coefficient (0.974). The researchers attempted to act scientifically and standardly in the field of scale production during the operation procedure; thus, that the method used in designing the EHLSQ could have the adequate assurance to design scales. Besides, the researchers tried to eliminate the previous scales' faults in assessing lifestyle while designing the EHLSQ.

\section{Ethical Considerations}

\section{Compliance with ethical guidelines}

All ethical principles were considered in this article. The participants were informed about the purpose of the research and its implementation stages; they were also assured about the confidentiality of their information; Moreover, They were allowed to leave the study whenever they wish, and if desired, the results of the research would be available to them.

Funding

The present paper was extracted from the $\mathrm{PhD}$. thesis the first author, Department of Health Management and Economics, School of Public Health, Tehran University of Medical Sciences.

\section{Authors' contributions}

Design and conceptualization: Fereshteh Farzianpour, Morad Esmaeil Zali; Methodology and data analysis: Morad Esmaeil Zali, Abbas Rahimi Foroushani; Supervision and final writing: Morad Esmaeil Zali, Mohammad Arab, Fereshteh Farzianpour.

\section{Conflicts of interest}

The authors declared no conflict of interest. 
This Page Intentionally Left Blank 


\title{
طراحى و اعتبارسنجى يرسشنامه سبك زندكَى سالم در بين سالمندان ايرانى گَيرنده خدمات

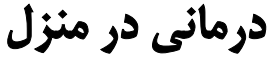

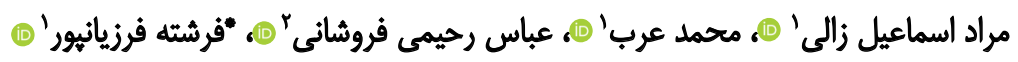 \\ I. كووه علوم مديريت و اقتصاد سلامت، دانشكده بهداشت، دانشكاه علوم يزشكى تهران، تهران، ايران

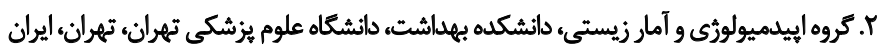

\begin{abstract}
(1)

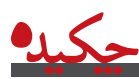

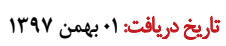

تاريخ هذيرش: بـ تير

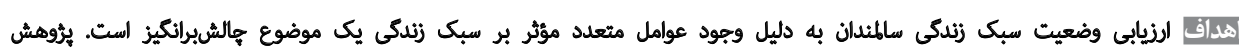

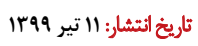

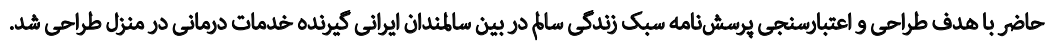

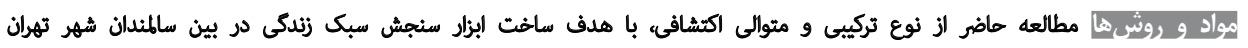

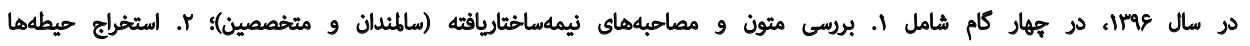

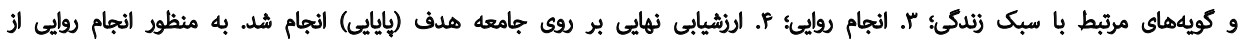

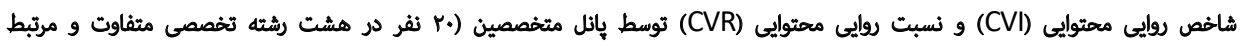

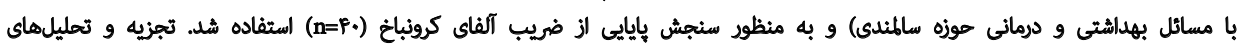

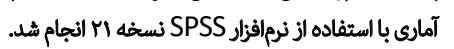

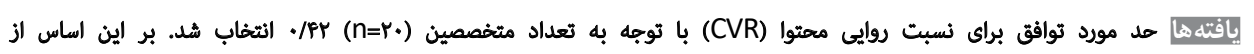

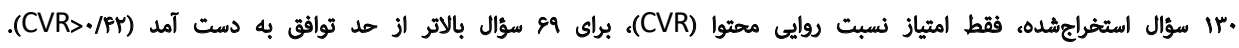

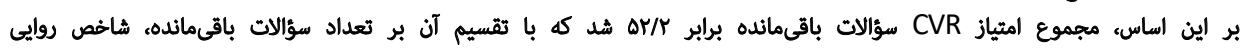

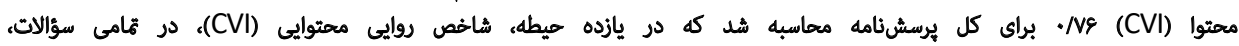

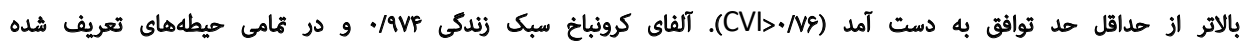
بالاتر از N/ بود.

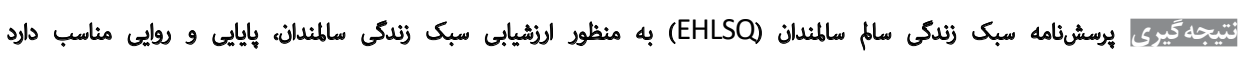

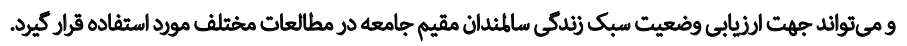

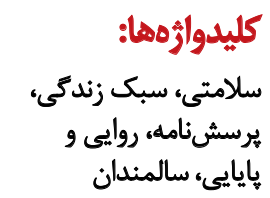

كليدوأرها:

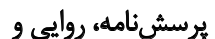

هايايايى، سالمندان
\end{abstract}

اين اساس با افزايش سن، سلامت افراد رو به افول كذاشته و بار

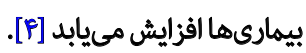

در حال حاضر، جامعه ايران به عنوان يك كشور در حال هال

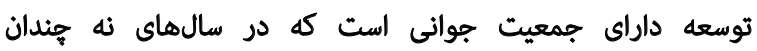

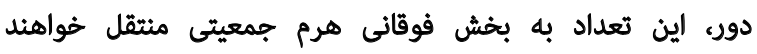

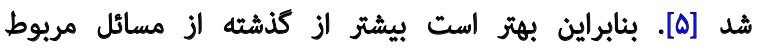

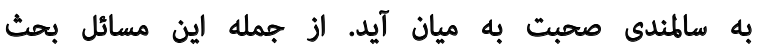

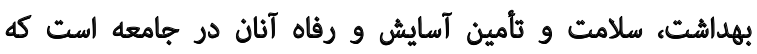

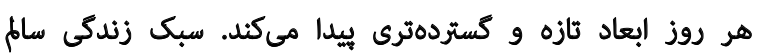

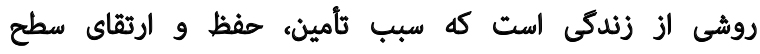

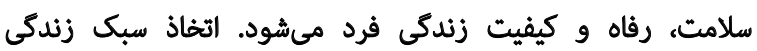

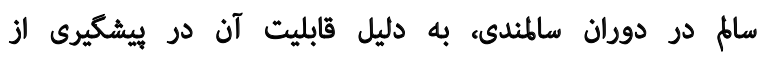

مقدمه

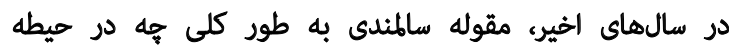

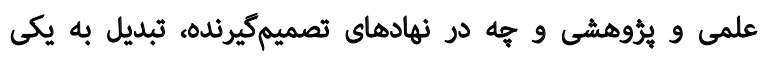

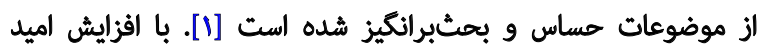

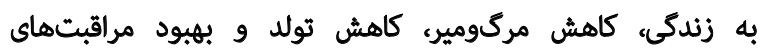

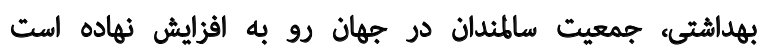

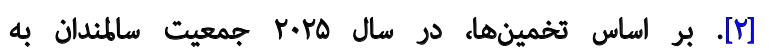

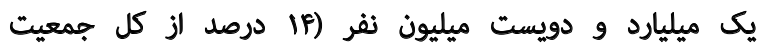

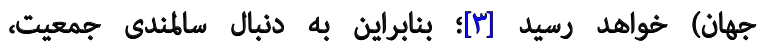

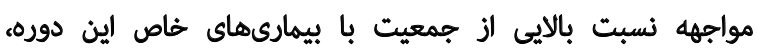

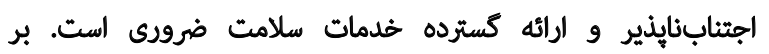


دوصدد بودنده با النجام بررسى جامع متون و مطالعات بيشين

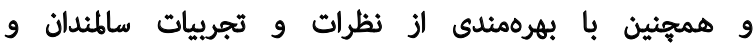

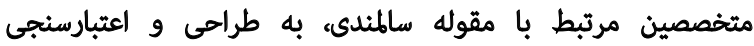

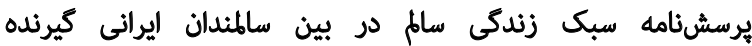

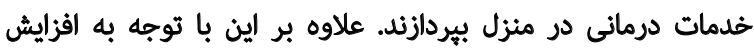

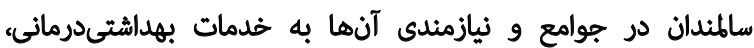

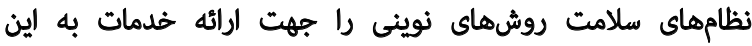

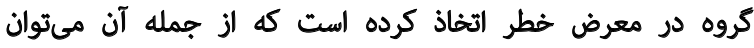

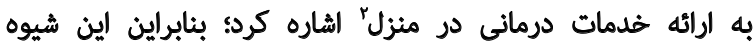

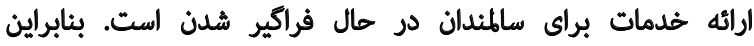

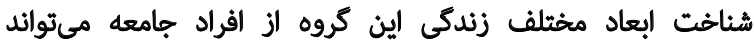

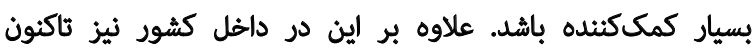

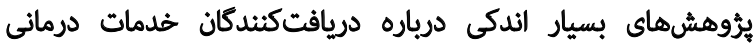

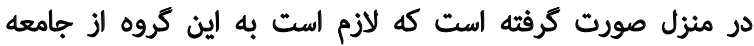

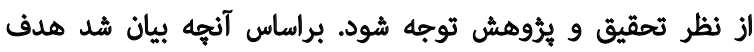

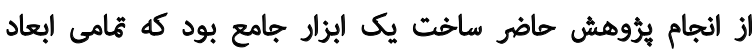
سبك زندكى را متناسب با مفهوم نظرى آن شامل شود.

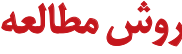

مطالعه حاضر الز نوع تركيبي و مثوالى اكتشافى با هدف طراحى،

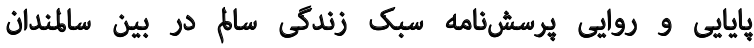

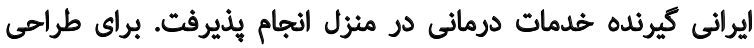

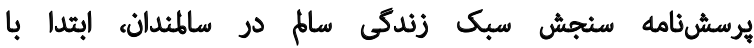
بروسى كتب، مقالات و يرسشنامههاى مشابه در زئه زمينه سبك

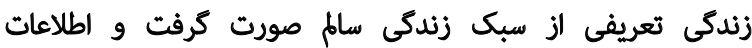

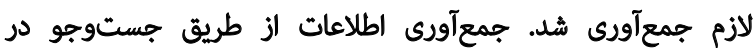

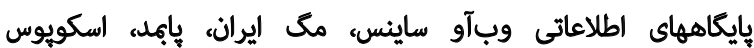

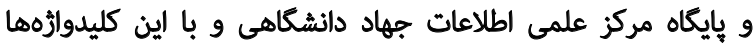

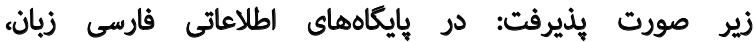

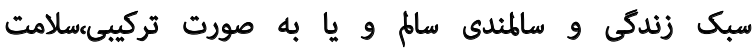

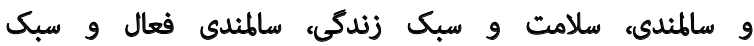

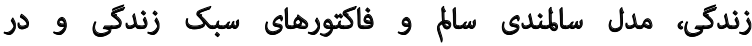

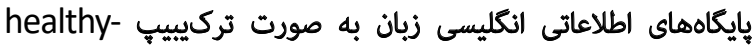
aging ،health and lifestyle ،elderly ، Health ،lifestyle Health and Aging Active Aging

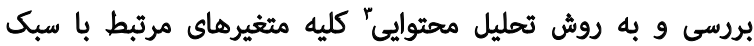

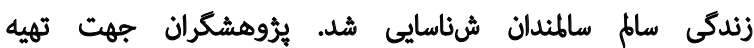

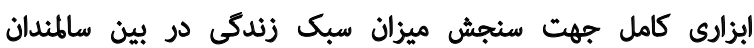

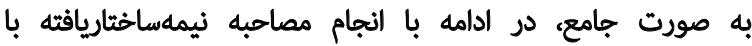

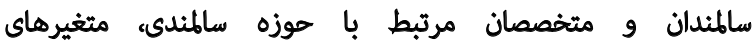

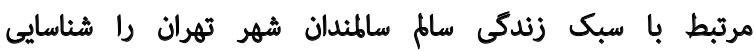

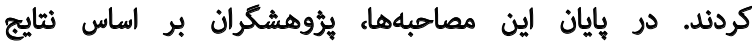

2. Home care

3. Content analysis
بيماريها، ارتقاى سطح كيفيت زندكى، افزايش اميد به زندكى و يهبود سلامت جسم و روان اهميت بسيارى دارد [عاريت.

سبك زندكى الكوهايى از رفتار مرتبط با سلامت است و متكى

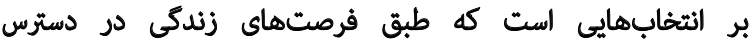
افراد است. اين انتخابها براي فهونه شامل تصميم براى سيكار

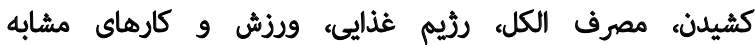

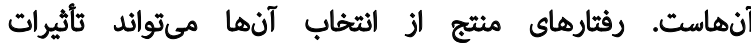

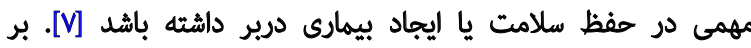

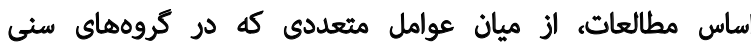

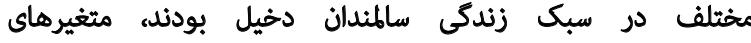

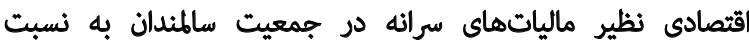

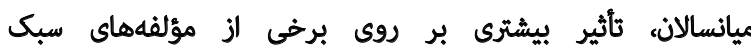

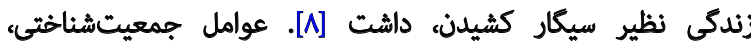

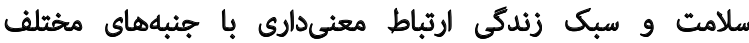

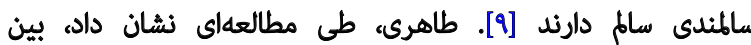

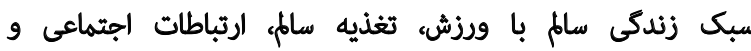
ييشكيرى ارتباطى مستقيم و بين سبك زندكى ودئ و استرس رابطه

معكوس معنى دارى وجود دارد [11]

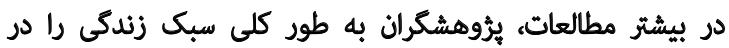

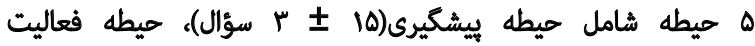

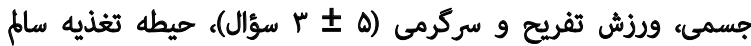

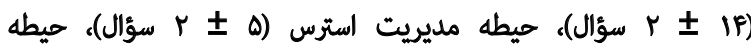

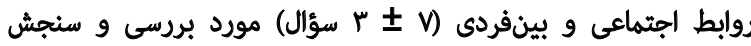

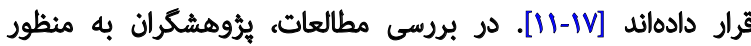

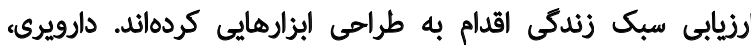

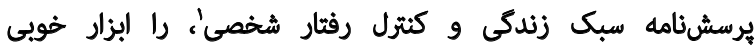

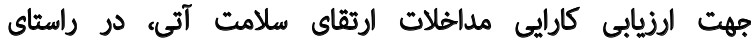

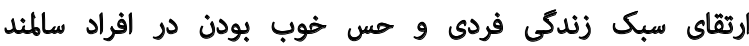

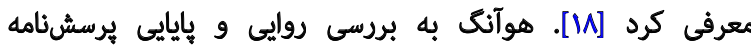

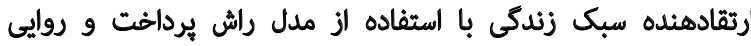

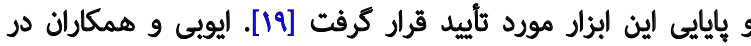

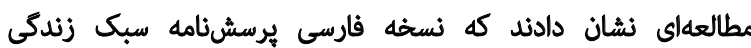

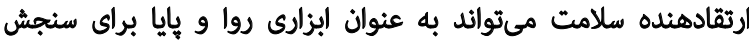

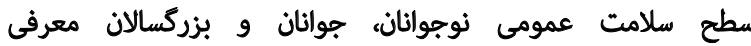

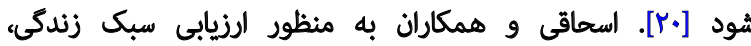

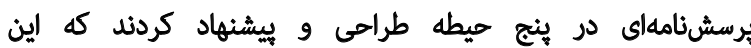

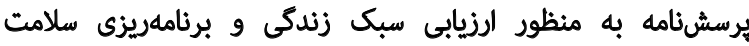

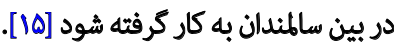

با وجود مطالعات انجامشده، به دليل افزايش مؤلفههاى مؤثر

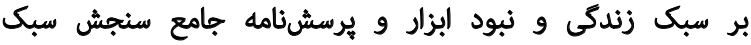

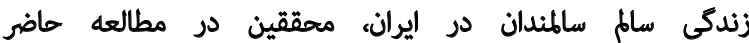

1. Healthy Lifestyle and Personal Control Questionnaire (HLPCQ) 
جدول ا. تعيين مقدار مورد توافق CVR برحسب تعداد افراد يثل متخصصين

\begin{tabular}{|c|c|c|c|}
\hline حداقل مقدار روايى & تعداد افراد ينل متخصصين & حداقل مقدار روايى & تعداد افراد هيثل متخصصين \\
\hline.$/ 4 q$ & 10 &.$/ 99$ & $\Delta$ \\
\hline.$/ 4 t$ & $r+$ &.$/ 99$ & $\varepsilon$ \\
\hline.$/ M V$ & $r \Delta$ & .199 & $\checkmark$ \\
\hline.$/ \pi n$ & r. & . /AA & $A$ \\
\hline \multirow[t]{2}{*}{.$/ 4 q$} & f. &.$/ \mathrm{NA}$ & 9 \\
\hline & & . IRT & 1. \\
\hline
\end{tabular}

记

شد. براى بررسى روايى محتوايى به شكل كمى، الز دو ضريب محتي

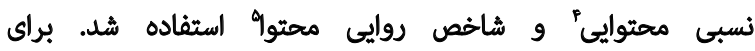

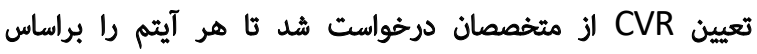

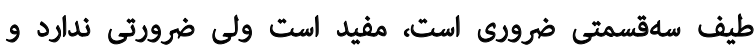

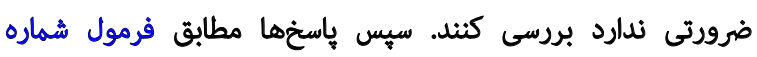

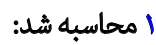

$$
C V R=\frac{n_{E}-\frac{N}{r}}{\frac{N}{r}}
$$

در اين رابطه nE تعداد متخصصانى الست كه به كزينه "ضرورى"

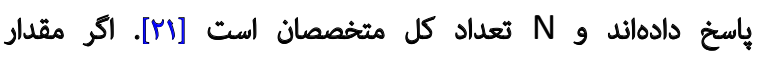
محاسبهشده از مقدار مورد توافق بزركتر باشد اعتبار محتواى آن آن

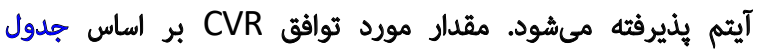

$$
\text { شماره ا تعيين شد. }
$$

براساس جدول شماره 1 و با توجه به اينكه تعداد متخصصان

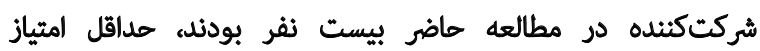

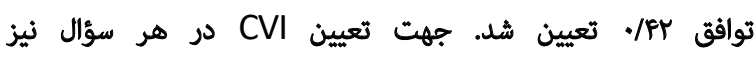

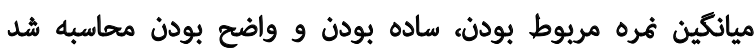

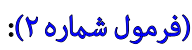

\section{.r}

$$
C V l=\frac{n}{N}
$$

در اين رابطه n تعداد متخصصانى است كه به كزينهای كاملاً

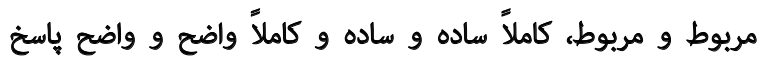

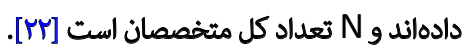

بنابراين در هنكام تعيين امتياز توافق براى مريوط بودن هر

4. Content Validity Ratio (CVR)

5. Content Validity Index (CVI)
بهدستآمده الز مطالعات متون و مصاحبه با سالمندان ابزار

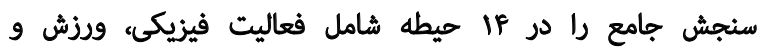

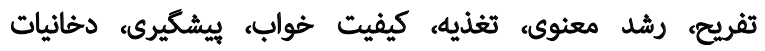

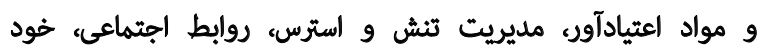

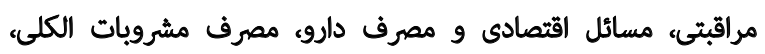

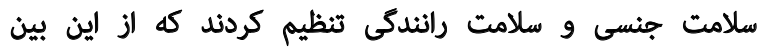
دو حيطه مشرويات الكلى و سلامت ولامت جنسى بله بيشنهاد كميته

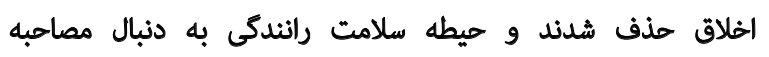

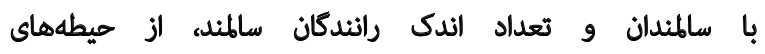

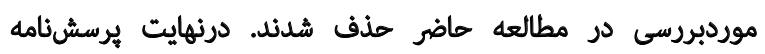

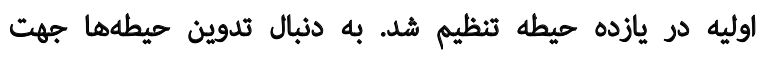

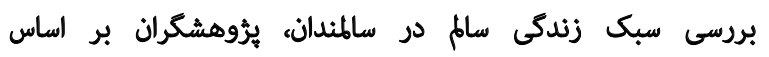
بروسى متون علمى داخلى و خارجى اقدام به استخراج كويهها

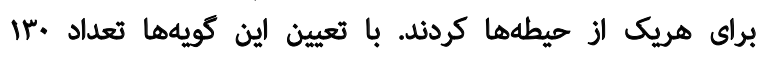

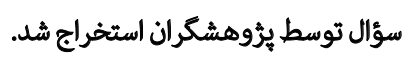

درنهايت كليه متغيرهاى الستخراجشده الز بروسى متون،

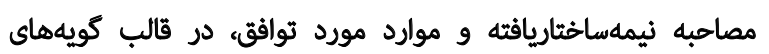

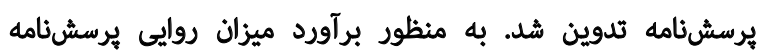

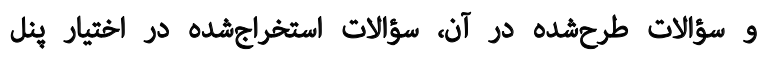

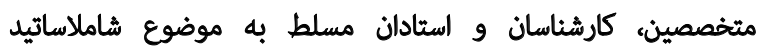

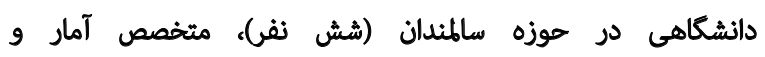

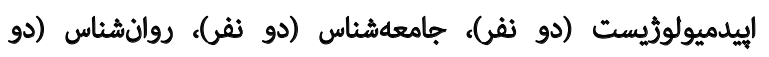

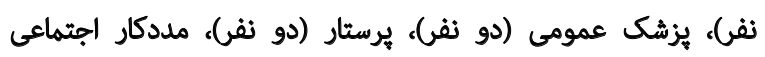

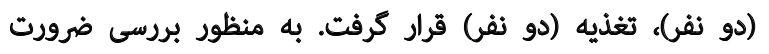

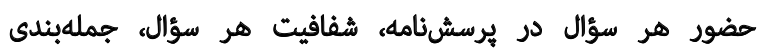

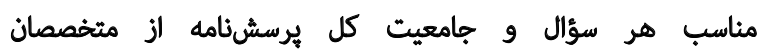

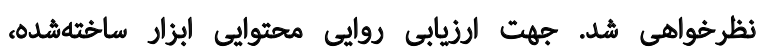

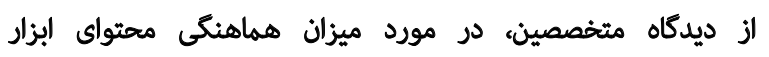

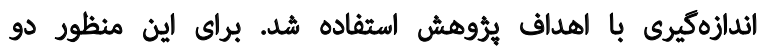

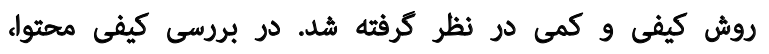

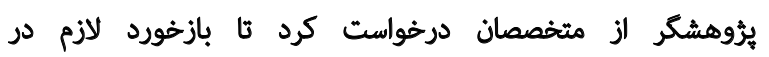

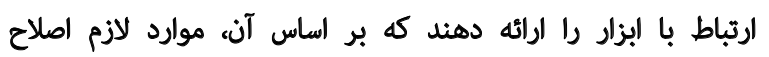


امثيازات تعريفشده در طيف ليكرت ابزار طراحىشده، به طور

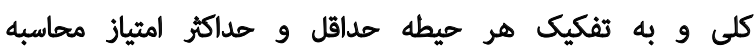

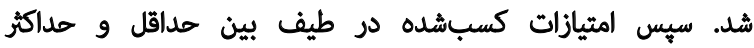

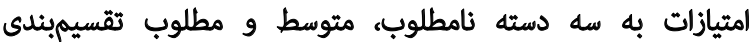

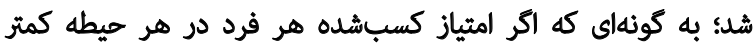

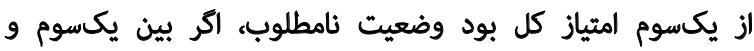

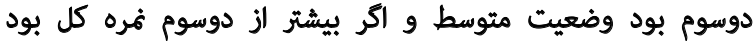
وضعيت مطلوب ثتريث مي شيد.

ياقتهها

حد مورد توافق براى نسبت روايى محتوا (CVR) با توجه

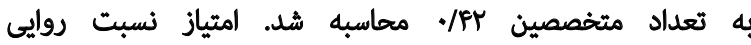
محتوا (CVR) براى هر سؤال در حيطههاى مذكور محاسبه شد.

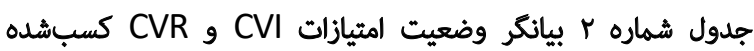
هر سؤال به تفكيك هر حيطه است. بر اين اساس دراس در حيطه فعاليت فيزيكى، ورزش و تفريح، نسبت روايى محتوايى (CVR)

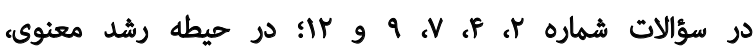

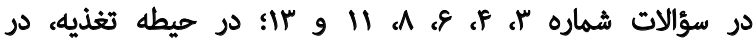

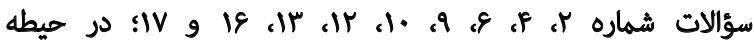

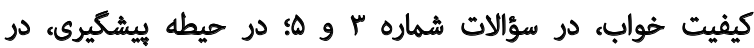

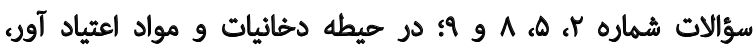

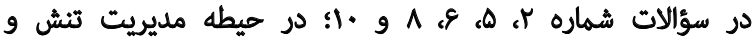

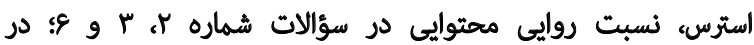

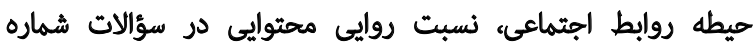

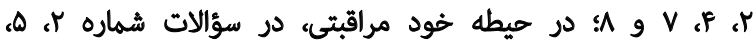

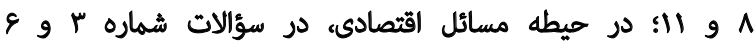

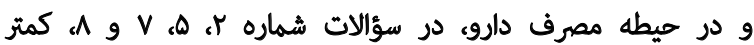

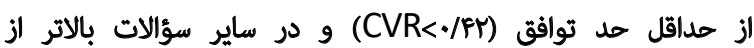
حداقل حد تواقق به دست آمد (CVR>-FT)

با در نظر كرفتن امتياز CVR، سؤالاتى كه امثياز آنها كمتر از

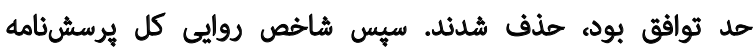

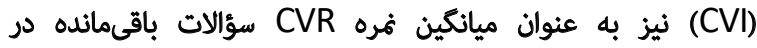

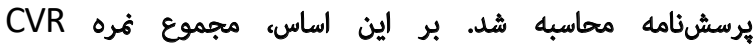

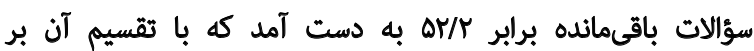

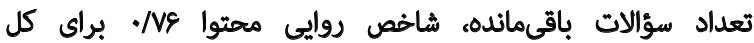

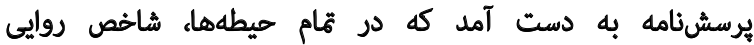

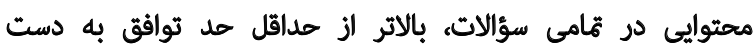
آمد (CVl>·/N9) (جدول شماره r).

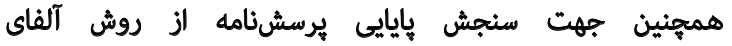

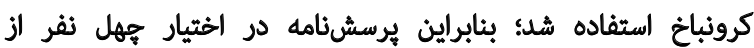

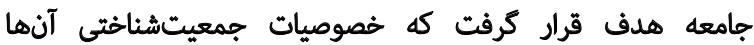

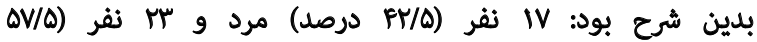
درصد) زن بودند. ميانكين و انحراف معيار سن شركت بنندكان
سؤال، ياسخهاى كاملاً مريوط و مريوط، در هنكام تعيين نمره

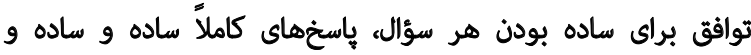

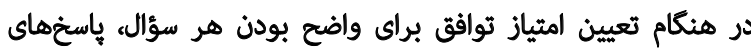

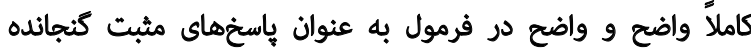

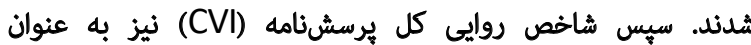

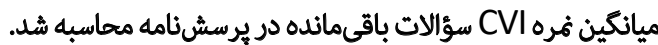

به دنبال اعمال نظر و اصلاحات خبركان و استادان، محققين

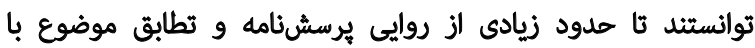

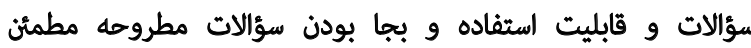

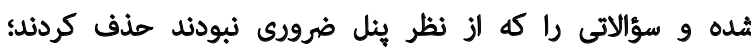

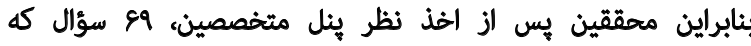

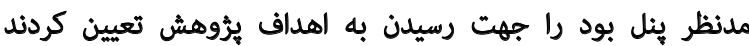

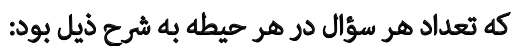
حيطه فيزيكى، ورزش و تفريح (هشت سؤال)، حيطه رشد معنوى (هشت سؤال)، حيطه تغذيه (ده سؤال)، حيطه كيفيت (هورئ

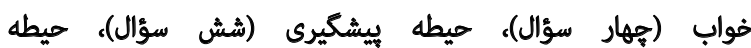

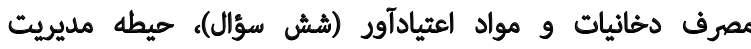

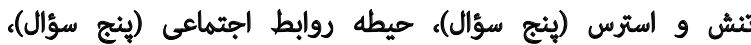
حيطه خودمراقبتى (هفت سؤال)، حيطه مسائل اقتصادى (ينج)

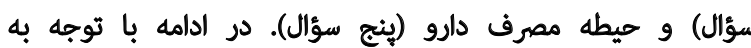

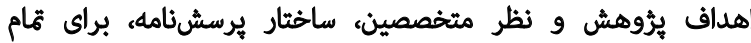

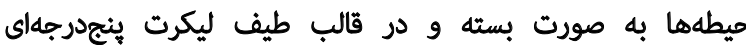

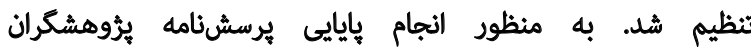

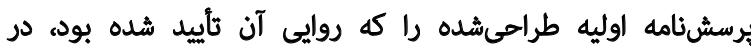

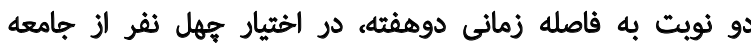

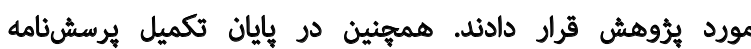

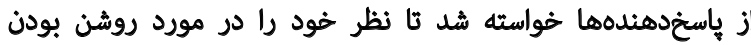

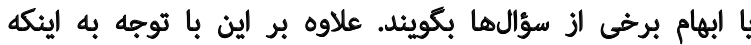

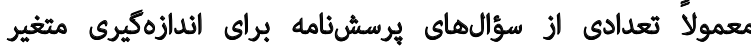

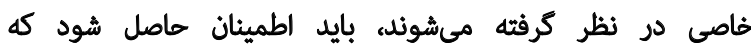

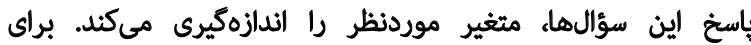

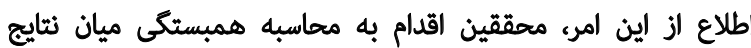
حاصلشده از ياسخهاى افراد به هر سؤال و ثمرات آنها كردند. ضريب همبستكى بالا ارتباط زياد ميان آنهه هر سؤال اندازه

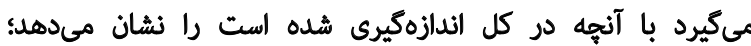

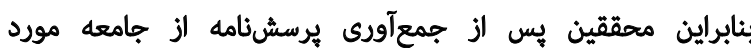

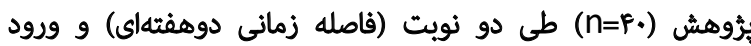

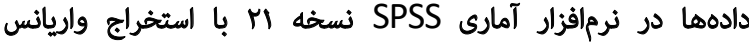

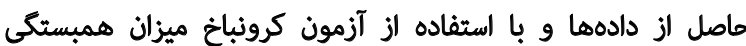

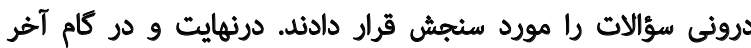

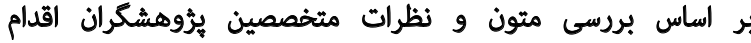

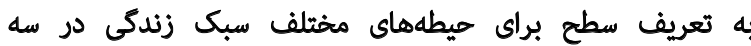
وضعيت مطلوب، متوسط و نامطلوب كردند. بدين منظور بر بر اساس 


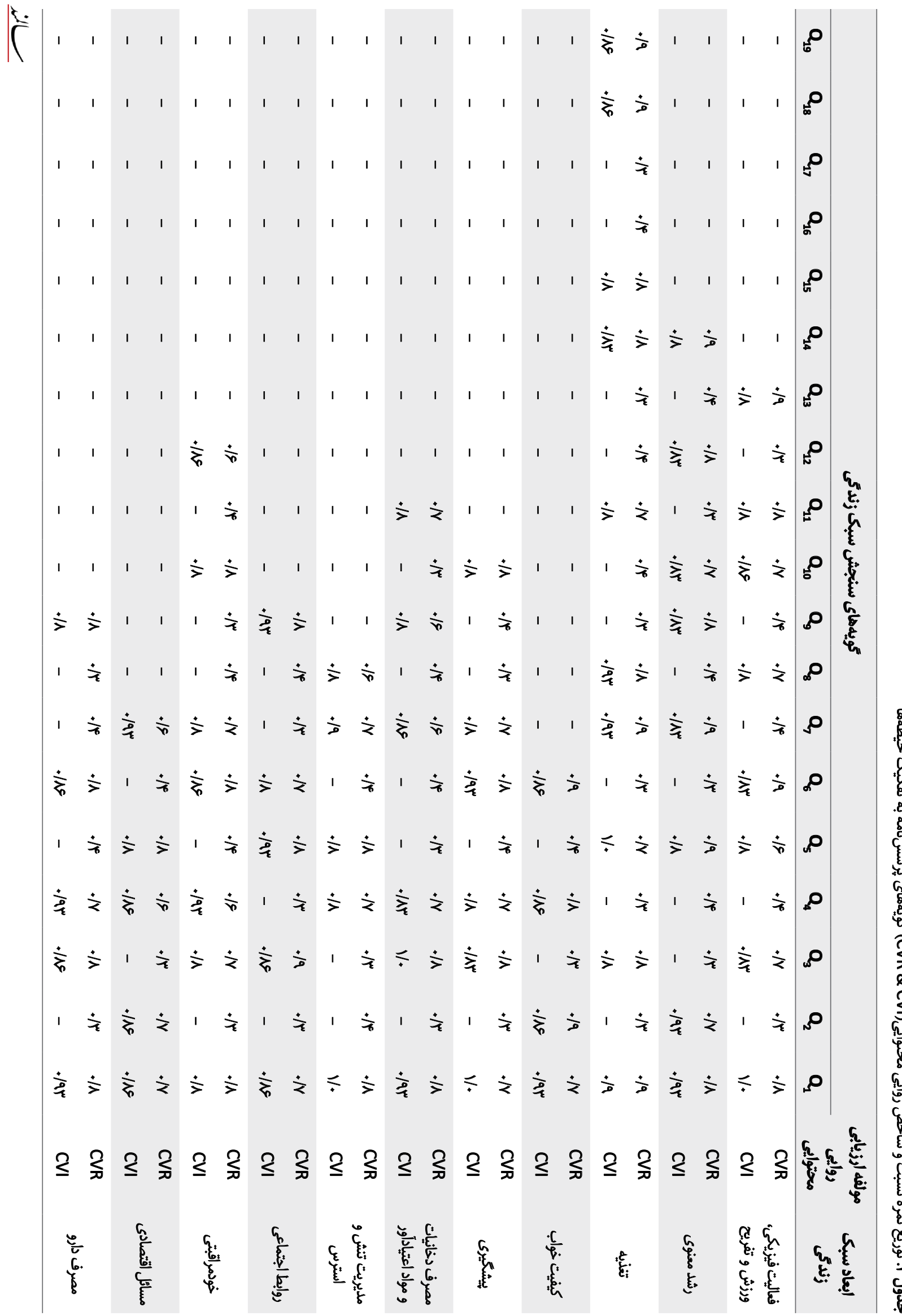

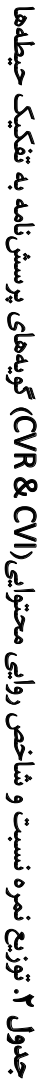


جدول ب. توزيع خصوصيات جمعيتشناختى سالمندان موردمطالعه

\begin{tabular}{|c|c|c|}
\hline \multicolumn{2}{|c|}{ ميانكين \$انحراف معيار / فراوانى (درصد) } & مثغير \\
\hline \multicolumn{2}{|c|}{$V \lambda \pm \lambda$} & سن، سال \\
\hline IV $(F T / \Delta)$ & هرد & \multirow[b]{2}{*}{ جنسيت } \\
\hline$M(\Delta V / \Delta)$ & & \\
\hline$r(\Delta)$ & هجرد & \multirow{4}{*}{ 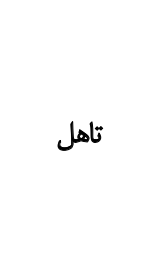 } \\
\hline$M(\varepsilon \cdot)$ & مثأهل & \\
\hline$P(1)$. & جلاشله & \\
\hline $1 \cdot(Y \Delta)$ & همسر فوتشده & \\
\hline$\Delta(I Y / \Delta)$ & بى سواد & \multirow{4}{*}{ تحصيلات } \\
\hline$\|(T V / \Delta)$ & ابتدايي & \\
\hline $18(f \cdot)$ & دييلم & \\
\hline$\Lambda\left(r_{0}\right)$ & دانشكاهى & \\
\hline$\Delta(M / \Delta)$ & شاغل & \multirow{4}{*}{ وضعيت شغلى } \\
\hline$M(\Delta V / \Delta)$ & بازتشستئه & \\
\hline $1 \cdot(T \Delta)$ & بيكار & \\
\hline$r(\Delta)$ & ساير & \\
\hline
\end{tabular}

次

جدول P. نتايج معيار آلفاي كرونباخ برسشنامه

\begin{tabular}{|c|c|c|}
\hline ضريب ألفاى كرونباخ (Alpha>+/V) & تعداد سؤالات & متخيرها \\
\hline.$/ 9 \cdot 1$ & $\wedge$ & فعاليت فيزيكمي \\
\hline ./QTA & $\Lambda$ & رشلد معنوى \\
\hline . /apr & 1. & تغذيه \\
\hline.$/ 9 F$ & f & كيفيت خُواب \\
\hline -/Asq & $\varepsilon$ & ييشكيرى \\
\hline.$/ 911$ & 8 & دخانيات و مواد اعتيادآور \\
\hline.$/ 911$ & $\Delta$ & مديريت تنش و استرس \\
\hline.$/ 119$ & a & روابط اجتماعى \\
\hline$\cdot / A \Delta \mid$ & $\checkmark$ & خودمراقبتى \\
\hline . / 974 & $\Delta$ & مسائل اقتصادى \\
\hline.$/ 970$ & $\Delta$ & مصرف دارو \\
\hline.$/ 97$ & 99 & سبك زندكى \\
\hline
\end{tabular}

U 
جدول ه. سطوح تعريف شده براي وضعيت سبك زندكى به تفكيك هر حيطه

\begin{tabular}{|c|c|c|c|c|c|c|c|}
\hline وضعيث مطلوب & وضعيت متوسط & وضعيت نامطلوب & هداكثر & فمرهاقل & سؤالات & عنوان حيطه & رديف \\
\hline$r \cdot<x<r q / \pi$ & $r q / m r<x<11$ & WeV $<x<1$ & $r$ & $\wedge$ & $\wedge$ & فعاليت فيزيكى & 1 \\
\hline$r \cdot<x<r q / r f$ & $r q / r F<x<11$ & $W E V<x<1$ & f. & $\wedge$ & $\wedge$ & رشد معنوى & r \\
\hline$\Delta .<X<r \& / \& V$ & $r g / \& V<x<m / M r$ & $M r / m<x<1$ & ه. & 1. & 1. & تغذيه & $r$ \\
\hline$Y \cdot<X<\mid f / e x$ & $\mid f / g r<x<q / m$ & $9 / \pi m<x<f$ & r. & f & f & كيفيت خواب & p \\
\hline$r \cdot<x<r$ & $m<x<1 f$ & if $<x<8$ & r. & 8 & 8 & ييشكيرى & $\Delta$ \\
\hline$r \cdot<x<r$ & $M r<x<$ If & if $<x<8$ & $r+$ & 8 & 8 & دخانيات و مواد اعتيادآور & $\varepsilon$ \\
\hline$r \Delta<x<W \pi r$ & $W M T<x<11 / F V$ & $11 / 8 x<x<0$ & ro & $ه$ & $\Delta$ & مديريت تنش و استرس & $\checkmark$ \\
\hline$r \Delta<x<W M r$ & $W / 4<x<11 / 9 V$ & $11 / 8 x<x<0$ & ro & $\Delta$ & $\Delta$ & روابط اجتماعى & $\Lambda$ \\
\hline$r \Delta r \Delta<x<18 V$ & $r \Delta / \& V<x<1 \& / M$ & $1 \& / \pi<x<v$ & ro & 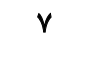 & $\gamma$ & حُودمراقبتئي & 9 \\
\hline$r \Delta<X<W N T$ & $W M T r<x<W / F V$ & WIEV $<x<\Delta$ & ro & $\Delta$ & $ه$ & مسائل اقتصادى & 1. \\
\hline$r \Delta<x<W M r$ & $W / r r<x<11 / e r$ & $11 / e V<x<0$ & ro & $\Delta$ & $\Delta$ & مصرف دارو & 11 \\
\hline$M T \Delta<x<r \Delta H^{H}$ & $\operatorname{rat}^{\mu}<x<|9|$ & $|q|<x<q q$ & MTa & $9 q$ & eq & نمره كلى سبك زندكى & ir \\
\hline
\end{tabular}

L

سبك زندكى سامل در بين سالمندان ايراني (EHLSQ)' كيرنده

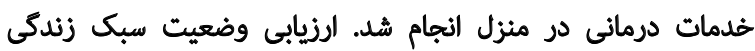

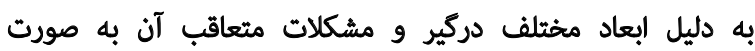

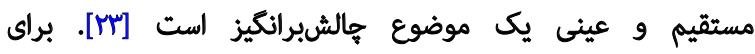

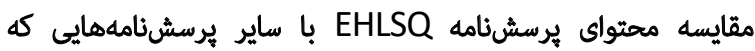

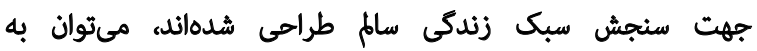

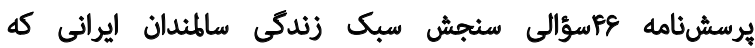
توسط السحاقي و همكاران [10] طراحي شده الست، الشاره كرد.

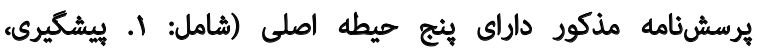

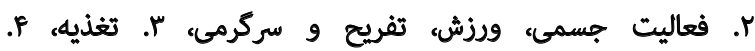

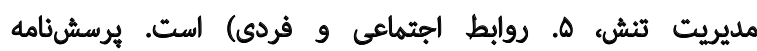

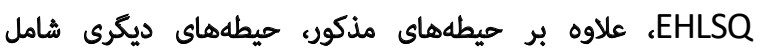
حيطه رشد معنوى، حيطه كيفيت خواب، حيطه مصرف دخان مخانيات

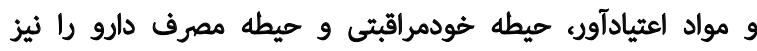
مورد ارزيابي قرار داده است. مواديت.

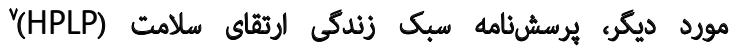

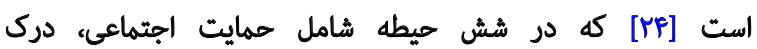

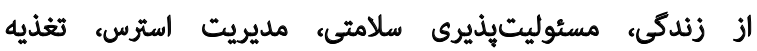

6. Elderly'S Healthy Lifestyle Questionnaire

7. Health Promoting Lifestyle Profile
سال بود. دو نفر (ه درصد) مجرد، MA VA

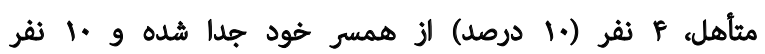

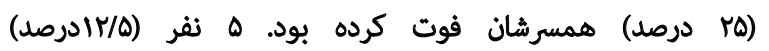

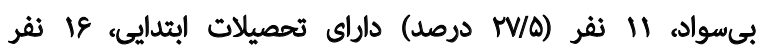

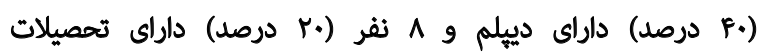

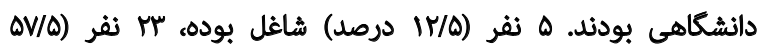

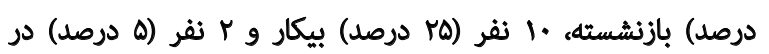

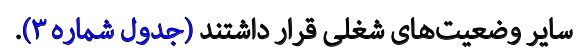
بر اساس يافتههاى جدول شماره fo آلفاى كرونباخ ميزان

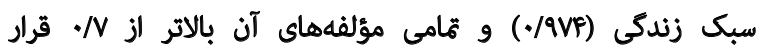
دارد.

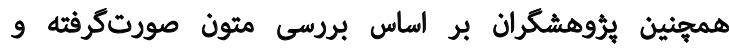

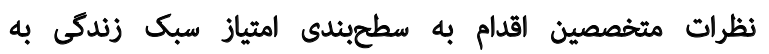

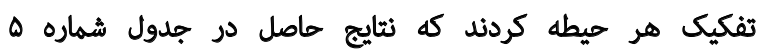

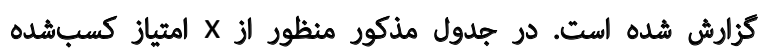
براى هر فرد است. ث يُروهش حاضر با هدف، طراحى، هايايى و روايى يرسشنامه 
شرايط فرهنكى داخل كشور بهتر از ثمونهاي خارجى مىثواند

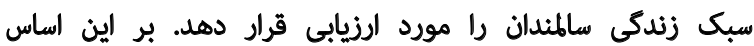

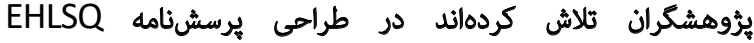

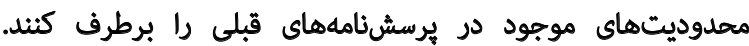

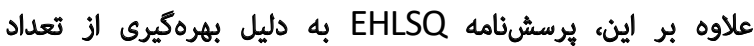
متخصصين بيشتر (بيست نف) و تنوع در تخصص (هشت رشته

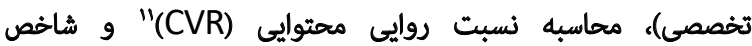

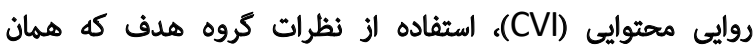

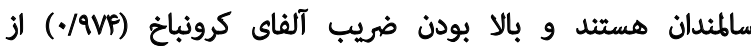

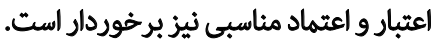

\section{نتيجديرىنهايي}

علاوه بر جامعيت محتوا و روايى و هايايى مناسب، ئوهشكران سعى كردند در روند اجرا نيز به صورت علمى و الستاندارد در

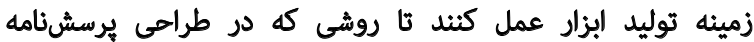

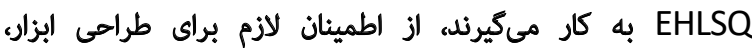

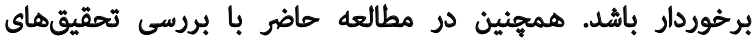

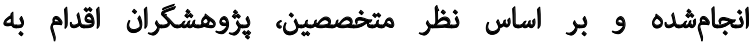
سطعبندى المثياز سبك زندكى كردند (جدول شهاره هان). علاوه

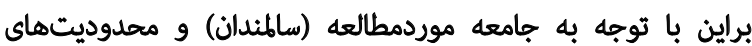

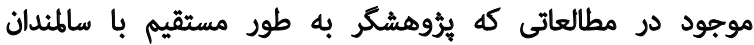

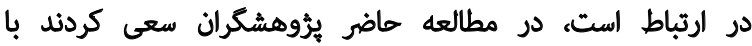

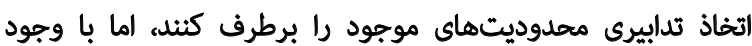

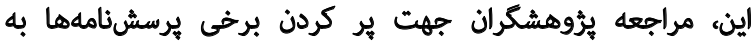

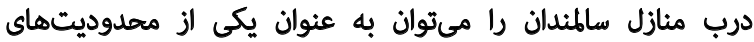

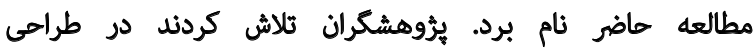

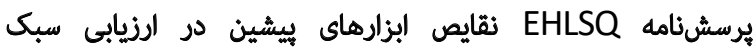

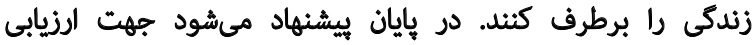

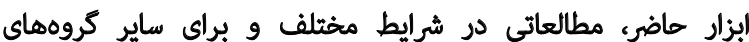
سنى انجام يذيرد.

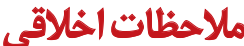

\section{ييروى از اصول اخلاق يثوهش}

همه الصول الغلاقى در اين مقاله رعايت شده است. شركت

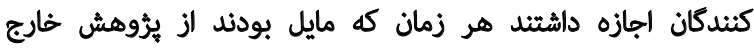

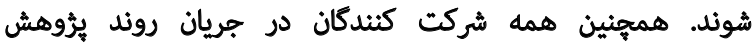

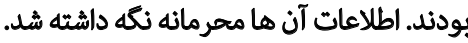

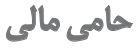

ايـن مقالـه بركرفتـهـ از بايـان نامـه دكـتز آقـاى مــراد

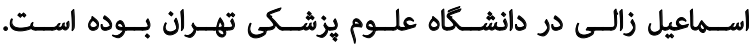

11. Content Validity Ratio
مناسب و فعاليت جسمى طراحى شده است. ايوبى و همكاران

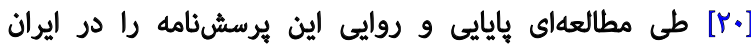

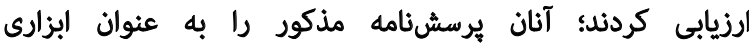

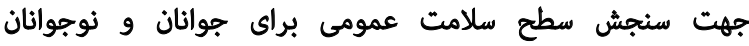

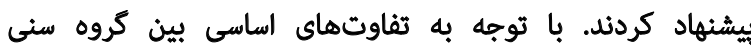
جوان و سالمند، به نظر تهىرسد يرسشنامه مذكور بتواند قابليت ارزيابى سبك زندكى براي ساملندان را دارا باشد.

يرسشنامه سبك زندكى و كنترل رفتار شخصى (HLPCQ)؛

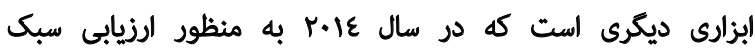

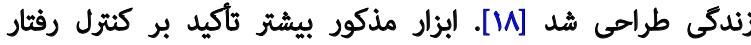
شخصى داشته و حيطه تغذيه در آن بيشتر مورد توجه بودئ بوده است.

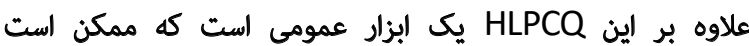

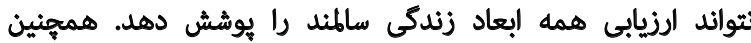

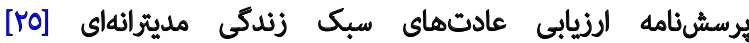

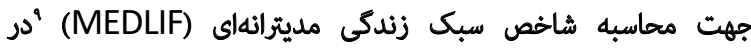

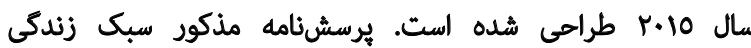

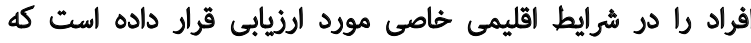

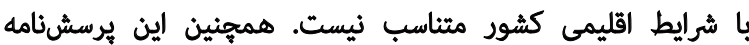

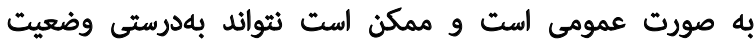

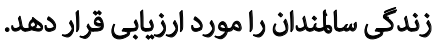

يرسشنامه سبك زندكى الرتقادهئده سلامت نسخه (HPLP-II)

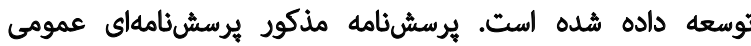

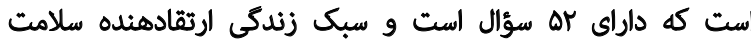

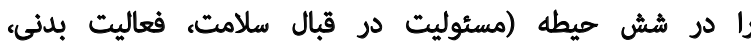
تغذيه، رشد معنوى، روابط بينفردى و مديريت استزس) مورد

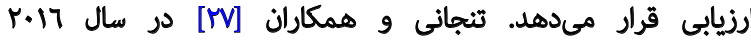

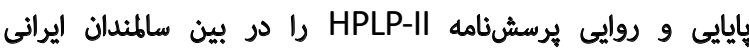

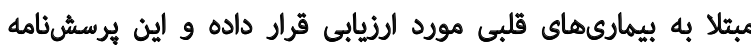
را ابزارى مناسب براي ارزيابى رفتارهاى الرتقادهنده سلامت بتايت

سالمندان ايرائي دانستند.

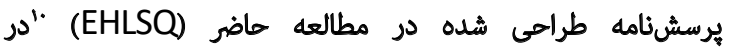

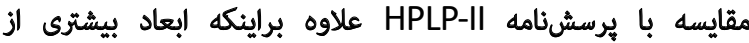

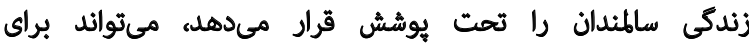

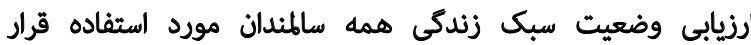

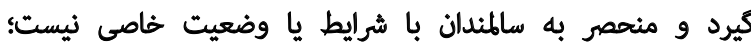

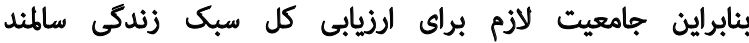

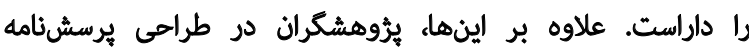
EHLSQ

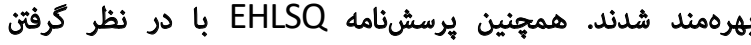

8. The Healthy Lifestyle and Personal Control Questionnaire 9. the MEDiterranean LIFEstyle index

10. Elderly's healthy lifestyle questionnaire 


$$
\text { مشاركت نويسندكّان }
$$

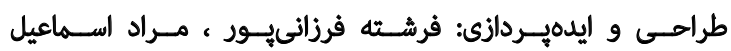

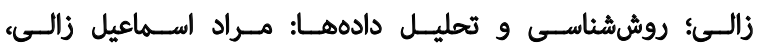

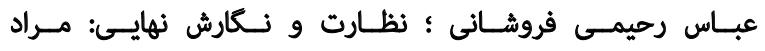

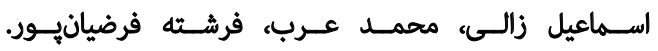

$$
\text { ثنعارض مئاقع }
$$

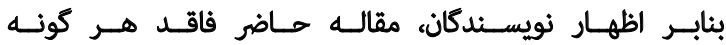
تعـارض منافـع بـوده اسـت نيست 


\section{References}

[1] Mohammadi M, Mohammadi Shahboulaghi F, Shati M, Khanke HR. [The validity and reliability of the Persian version of the device to identify older people at risk for readmission in emergency centers (Persian)]. Nursing Journal of Vulnerable. 2016; 3(8):48-58. http:/ / njv.bpums.ac.ir/ article-1-740-en.html

[2] Park SH, Han KS, Kang CB. Effects of exercise programs on depressive symptoms, quality of life and self-esteem in older people: A systematic review of randomized controlled trials. Applied Nursing Research. 2014; 27(4):219-26. [DOI:10.1016/j.apnr.2014.01.004] [PMID]

[3] Mohammadzadeh A, Dolatshahy B, Mohammadkhani P. [The effects of integrative reminiscence therapy on signs and symptoms of depression in the elderly (Persian)]. Iranian Journal of Ageing. 2011; 6(1):23-8. http://salmandj. uswr.ac.ir/article-1-373-en.html

[4] Imani ADS, Azizi Zeinalhajlou A. [Population aging and burden of diseases (Persian)]. Depiction of Health. 2015; 6(2):54-61. https://doh.tbzmed.ac.ir/Article/doh-125

[5] Statistical Center of Iran. Selected findings of the 2016 national population and housing census. Tehran: Statistical Center of Iran; 2018. https:/ / www.amar.org.ir/Portals/1/ census/2016/Census_2016_Selected_Findings.pdf

[6] Hekmatpou D, Shamsi M, Zamani M. [The effect of healthy lifestyle educational programs on the quality of life of the elderly in Arak (Persian)]. Journal of Arak University of Medical Sciences. 2013; 16(72):1-11. http:/ /jams.arakmu. ac.ir/article-1-2024-en.html

[7] Ghasemi V, Rabani R, Rabbani A, Alizadeh MB. [Structural and capital determinants of health-oriented lifestyle (Persian)]. Journal of Faculty of Literature and Humanities Kharazmi. 2009; 1387(63):181-213. https://jspi.khu.ac.ir/ article-1-229-fa.html

[8] Son KY, Park SM, Lee J, Kim CY. Difference in adherence to and influencing factors of a healthy lifestyle between middle-aged and elderly people in Korea: A multilevel analysis. Geriatrics \& Gerontology International. 2015; 15(6):778-88. [DOI:10.1111/ggi.12335] [PMID]

[9] Kendig H, Browning CJ, Thomas SA, Wells Y. Health, lifestyle, and gender influences on aging well: An Australian longitudinal analysis to guide health promotion. Frontiers in Public Health. 2014; 2(2):70. [DOI:10.3389/ fpubh.2014.00070] [PMID] [PMCID]

[10] Taheri M. The study of life style and demographic characteristics of iranian elderly. Journal of Sports Research. 2014; 1(3):45-8. https://ideas.repec.org/a/pkp/ josres/2014p45-48.html

[11] Movahedi M, Khamseh F, Ebadi A, Haji Amin Z, Navidian A. [Assessment of the lifestyle of the elderly in Tehran (Persian)]. Journal of Health Promotion Management. 2016; 5(3):51-9. http:/ /jhpm.ir/article-1-630-en.html

[12] Abbasi M, Daniali SSM, Hazrati M. Lifestyle of fallen elderly patients referred to Isfahan Hospitals. Iran Journal of Nursing. 2017; 30(107):20-31. [DOI:10.29252/ijn.30.107.20]

[13] Anahita Babak SD, Davari S, Aghdak P, Pirhaji O. Assessment of healthy lifestyle among elderly in Isfahan, Iran.
Journal of Isfahan Medical School. 2011; 29(149):64-74. https://b2n.ir/578878

[14] Fallah Mehrabadi E PM, Asadi S, Haghani H. [Lifestyle of elderly people with osteoporosis and its related factors (Persian)]. Iranian Journal of Ageing. 2017; 12( 2):132-45. [DOI:10.21859/sija-1202132]

[15] Eshaghi SR, Farajzadegan Z, Babak A. [Healty lifestyle assessment questionnaire in elderly: Translation, reliability and validity (Persian)]. Payesh. 2010; 9(1):91-9. http:// payeshjournal.ir/article-1-593-en.html

[16] Shamsadini Lori A, PourMohammadi K, Vida K, Ahmadi Kashkol S, PourAhmad MR. [A survey on the level of the elderly lifestyle in Shiraz (Persian)]. Journal of HealthBased Research. 2015; 1(1):75-84. http://hbrj.kmu.ac.ir/ article-1-35-en.html

[17] Mahmudi Gh, Niazazari K, Sanati T. [Evaluation of life style in the elderly (Persian)]. Journal of Health Breeze. 2013; 1(3):45-50. http://jfh.iausari.ac.ir/article_633408.html

[18] Darviri C, Alexopoulos EC, Artemiadis AK, Tigani X, Kraniotou C, Darvyri P, et al. The Healthy Lifestyle and Personal Control Questionnaire (HLPCQ): A novel tool for assessing self-empowerment through a constellation of daily activities. BMC Public Health. 2014; 14(1):995. [DOI:10.1186/1471-2458-14-995] [PMID] [PMCID]

[19] Hwang JE. Promoting healthy lifestyles with aging: Development and validation of the Health Enhancement Lifestyle Profile (HELP) using the Rasch measurement model. American Journal of Occupational Therapy. 2010; 64(5):78695. [DOI:10.5014/ajot.2010.09088] [PMID]

[20] Aubi E, Shadnoush M, Nazarzadeh M, Bidel Z, Ranaei A, Delpisheh A. [Translation and assessment of validity andreliability of the health-promoting lifestyle questionnaire, using factor analysis (Persian)]. Pejouhandeh. 2012; 17(3):114-20. https://www.academia.edu/21511928/

[21] Hajizadeh E, Asghari M. [Methods and statistical analyzes by looking at the research method in the biological sciences and health sciences (Persian)]. Tehran: University Jihad Publishing Organization; 2011. https://b2n.ir/425749

[22] Mohd Tajudin N, Puteh M, Mazlini A. Guiding principles to foster higher order thinking skills in teaching and learning of mathematics. International Journal of Engineering \& Technology. 2018; 7(4.15):195-9. [DOI:10.14419/ijet. v7i4.15.21445]

[23] Rodriguez Añez CR, Reis RS, Petroski EL. Brazilian version of a lifestyle questionnaire: Translation and validation for young adults. Arquivos Brasileiros de Cardiologia. 2008; 91(2):102-9. [DOI:10.1590/S0066-782X2008001400006] [PMID]

[24] Chen MY, Wang EK, Yang RJ, Liou YM. Adolescent health promotion scale: Development and psychometric testing. Public Health Nursing. 2005; 22(2):209-20. [DOI:10.1046/j.1525-1446.2003.20204.x]

[25] Sotos-Prieto M SBG, Bodega P, Pocock S, Mattei J Peñalvo JL. Validation of a questionnaire to measure overall Mediterranean lifestyle habits for research application: The MEDiterranean LIFEstyle Index (MEDLIFE). Nutricion Hospitalaria. 2015; 32(3):1153-63. http://scielo.isciii es/ scielo.php?pid=S0212-16112015000900026\&script=sci abstract\&tlng=en 
[26] Walker SN, Sechrist KR, Pender NJ. The health-promoting lifestyle profile: Development and psychometric characteristics. Nursing Research. 1987; 36(2):76-81. [DOI:10.1097/00006199-198703000-00002] [PMID]

[27] Tanjani PT, Azadbakht M, Garmaroudi G, Sahaf R, Fekrizadeh Z. Validity and reliability of health promoting lifestyle profile II in the Iranian elderly. International Journal of Preventive Medicine. 2016; 7:74. [DOI:10.4103/20087802.182731] [PMID] [PMCID] 